\title{
RESIDENCY IN PERSPECTIVE
}

\author{
James PARKhouse, M.A., M.D., F.F.A.H.C.S., D.A.*
}

\section{Two kinds of staff}

IN A HOSPITAL DEPARTMENT-and particularly in a specialty such as anaesthetics, where much of the routine work of the day is of a technical nature-it can be argued that there should be two types of men on the staff: an intellectual elite who attend meetings, write papers, and conduct research, and a hard core of good honest clinicians who do the work. What constitutes doing the work is a large question, and what may properly be regarded, in medicine, as an intellectual elite is a difficult one. I have expressed myself elsewhere about the dangers of a "two culture" philosophy, with confrontation between hospital and university, but in practice it is something we live with whether we like it or not; and since there is so much to be said about different grades and kinds of people, it is a good place to begin.

We need consultants who are consultants. Their function is to consult. But much of the work involved in looking after patients today is not of this order; much is technical and more will be tomorrow. Who is to do this work? We do not need a senior consultant to set up every intravenous infusion; it is an unjustifiable waste of our human resources to use him thus. But we do need somebody to do this and innumerable other humble tasks, with appropriate supervision. We have to think not of one or two but of many kinds of people on the "staff" of the hospital and the university.

\section{The resident and the work load}

In regard to competent assistance, there is clearly a difference between hospitals with residents and those without. The residents do, in fact, assist in many ways with the work of the hospital and its doctors; they are remunerated accordingly and they derive much benefit from the experience. There has been talk in Britain about using the trainee in hospital as a pair of hands, and with good reason, but it is important to emphasize the need for actual work and real clinical responsibility during training. The danger comes when the employment of residents is related solely to the service requirements of the hospital.

\section{Quality and quantity}

Approval of hospitals for residency training has long been based on what the hospital has to offer. So far so good; we must never forget that the primary consideration in deciding on the placing and the numbers of residents in a programme must be what the hospital can offer the resident, not what the resident can offer

- Postgraduate Dean of Medicine, University of Sheffield, England. Formerly Professor and Chairman of the Department of Anaesthetics, University of Manitoba, and Chief Anaesthetist, Winnipeg General Hospital. 
the hospital. But in practice there are other considerations: hospitals and hospital commissions, who foot the bill, feel entitled to see residents doing some work. There is also a status value in having residents, which may lead to a glossing over of some inadequacies in the facilities available. There is an even greater status value in being able to say that the residency posts in a department are all filled. It is also necessary to provide enough residency appointments in the country to accommodate the graduates who desire them, from home and abroad.

The present move towards university residency programmes, rather than individual hospital accreditations, has focused attention on the academic content and overall balance of the residency and this is very much to the good. At the same time, many departments are perhaps taking a second look at the foreign graduate - why he comes, where he intends to go, what his special needs are and what his place in the overall scheme of things should be. For both of these reasons we can expect a shift in emphasis in residency selection from quantity to quality.

\section{Who pays the piper?}

Hopeful as this may be for the brave new academic world we must beware of exchanging an uneasy marriage for a catastrophic divorce. Payment of residents by hospitals in return for hospital work may have clear disadvantages but it does put the emphasis in the right place-on clinical experience-and it is better than not paying residents at all. If residency programmes are henceforth to become a university affair it is reasonable to ask whether the universities should pay, at least in part, for the training programme. But the universities could well argue that they offer a valuable service in training residents, which is expensive enough already in terms of faculty time and effort, and for which they can expect no return, as the hospitals can, in the form of service now or later. In Britain, the National Health Service is the ultimate employer of trainees and trained specialists, and the cost of training, even in its academic aspects, is a legitimate longterm government investment. When private practice is the rule of the day we must acknowledge that the person who stands to gain from training is primarily the resident himself, and he can rarely afford to invest in his future to the extent of paying his own way. I see no easy answer to this dilemma except a sharing of responsibility.

\section{All chiefs and no Indians}

If we are to regard residency as a learning experience rather than a spell of junior hospital work, and if we are to explore means of remuneration which might relieve the resident of some of his obligation to the hospital, we must face again the question of who does the clinical work-and not just the same amount of work as before but more, because we can anticipate a much larger teaching commitment. Are we to place all hospitals in the present position of those without residents, where in effect all the medical work, whatever degree of skill and experience it requires, is done by specialists? Are we to introduce a second, nontraining grade, or are we, after all, to settle for two types of consultant? 


\section{The tradition of one to one}

The concept of the physician who assumes personal responsibility for his patient is time-honoured and noble. Inherent in the private practice of medicine is the belief that if a patient chooses to come to you, above all other doctors, then you alone must take care of him. In simple circumstances this is a precept which can be applied literally without too obvious a loss of efficiency. But in the complex setting of today's hospital practice it becomes increasingly hard to maintain a feeling of one-to-one relationship between patient and physician. A man sees a surgeon; the surgeon regards the man as his patient. The patient is admitted to hospital and needs extensive radiological investigation, followed by anaesthesia and surgery. Whose patient is he in the X-ray department, or when his blood pressure falls under anaesthesia? And when he needs treatment for a postoperative myocardial infarction, whose patient is he then? Are the radiologist, the anaesthetist, and the cardiologist all in some sense servants of the surgeon, or is the patient in fact being managed by a team? And if the team can include several specialists, may it not also include radiographers, recovery room nurses, ECG technicians, and perhaps medical assistants?

Much confusion and difficulty is generated by one-to-one thinking. To be sure there are problems, more evident in private practice than in a National Health Service, concerning what may properly be delegated to an assistant; but surely all modern medicine must be founded on the team management concept. We need true consultants because we must have true consultation between all those responsible for the patient's welfare. The lack of this is the greatest defect of our medical practice today.

It is naturally in the areas of most intensive patient care that the need for teamwork becomes most evident. The obvious difficulty of organizing an intensive care unit on a fee-for-service basis bears witness to this; but this is only the first crack in a system which is inherently inappropriate to the medicine of tomorrow. Certainly in anaesthesia it becomes increasingly apparent that the consultant, the assistant, the nurse, and the technician all have their place in the team. The question should not be "who is to give this anaesthetic?" but rather "how are all the patients actually and potentially at risk from unconsciousness and respiratory distress to be most efficiently cared for with the manpower resources available?" Not one to one but one to many, and many to one.

\section{Medical students and manpower}

Now that manpower has been mentioned it is appropriate to bring in the medical student. Many specialties, including my own, are obsessed by a recruitment problem. They see a shortage of specialists, for there is a shortage. They look for an answer through recruitment, and what better place to start than with the student? The feeling is that if the student can be exposed to anaesthesia, or whatever the specialty may be, as much as possible, if he can be shown what it has to offer, he may consider taking it up when he graduates. There is force in the argument, viewed pragmatically. It works. There is also perhaps more to be said for it in 
anaesthesia than in some other specialties because of the size of the problemanaesthesia in Britain is the largest single specialty in the National Health Service. But there are other considerations.

Medical students have many demands on their time and mental energy, and recruiting drives, however skilfully conducted, are not legitimate ones. There is, moreover, a limit to the total output of our medical schools. We cannot solve our manpower problem by doubling the number of doctors in the country overnight; we cannot solve it by robbing Peter to pay Paul. It may be that we have more potential specialists in some field than we need, and it is certain that better vocational guidance should be offered in the medical school with regard to more and less popular specialties; our best hope of resolving our difficulties lies in the most efficient deployment of the men and women that we have.

\section{The medical assistant}

Several experiments have been made in Britain, since the National Health Service began, with medical assistant grades. The first was the Senior Hospital Medical Officer, a trained doctor working in a hospital specialty but without the postgraduate qualifications of a consultant. There were several difficulties with the sHmo grade and it is hard to sum them up. It might be fair to say that the sHMo was too near to the consultant to be happy with his lot; many became consultants in the course of time and the grade declined. Recently, Medical Assistants were introduced, but here the name was unfortunate and the posts are not universally popular. Among the recommendations of the Royal Commission Report on Medical Education is again a proposal for two grades of non-trainee hospital doctor; this time to be called Consultant and Specialist. The intention here is that a Specialist will have security of tenure with the possibility of proceeding to Consultant rank after further graduate training if he so desires. How this will work remains to be seen. The important point is that after twenty years of experience, not by any means all favourable, and after very deep and careful thought, the conclusion has once again been reached that a second grade of some kind is necessary.

\section{The technical assistant}

We all try to think nowadays not in terms of medicine but in terms of the health sciences. We hear of medical auxiliaries and feldschers, and a host of technicians and nurse-technicians surrounds us. Nurses are university graduates, in increasing proportion; pharmacists are graduates. We don't know any more what a doctor really is, or what a nurse really is, or what properly constitutes the work they are supposed to do. Although some specialties, such as radiology, have employed technical help for many years practice varies widely in different countries: North America has inhalation therapy technicians which Britain does not; Britain has midwives; the United States and the Scandinavian countries have nurse anaesthetists.

This is not the place for a long discussion of a complex problem. It should be sufficient to emphasize the need for what is popularly called a critical reappraisal of some of our practices, and especially our prejudices in regard to the delegation 
of some of the traditional work of the doctor to trained assistants who do not necessarily have an M.D. degree. Better by far to do this than plan recruiting drives to get residents as pairs of hands.

\section{Training for what?}

A reappraisal of the role of the doctor presupposes a reappraisal of his training. Training is not an end in itself. It is not even a thing in itself. None of us is ever completely trained. Our purpose is to prepare people as well and as efficiently as we can for the responsibilities that they are to assume. At any time these responsibilities must in turn be commensurate with the attainment and ability of the person who assumes them. In looking at residency training and the resident's responsibilities, then, we must appreciate that we are looking at one phase of an extensive process-the process of maintaining an appropriate health service for the community.

Enough has been said already, and enough has been written elsewhere, to leave no doubt that we live in a world of change. Medicine advances with unprecedented speed and in unexpected directions. We can only meet this situation by preserving flexibility, in ourselves and in our training of the next generation. Preserving is an important word, for we should realize that people are born more flexible than we think. It is not so much a matter of creating open-mindedness and breadth of vision as of preventing its destruction through undergraduate indoctrination and narrow specialist training. The most important fact to carry with us through our educational programmes is that none of us received a training that is appropriate to what we do today and that whatever we teach our students will be obsolete tomorrow. Unless, that is, we teach them to think.

\section{The academic concept}

There is an old debate about the proper nature of medical education which is especially relevant to the graduate student: whether we are training anaesthetists and surgeons in a job-specific sense, as we train bus drivers, or whether we are educating people in the truly general spirit of the university. Hence the rather uncertain feeling as to whether graduate medical education is at all the proper concern of universities, or whether it should be a clinical vocational training.

It is clear that we need a compromise. We hear now that the job of the medical school is to produce undifferentiated or multipotent doctors. This implies emphasis on the academic concept of training the mind, rather than the vocational concept of training the hands. I think it is right, but I also think that in a world where new specialties are likely to arise while old ones decline we need many of our more highly trained graduates to be multipotent as well. What we really need is undifferentiated thinkers, who can apply their minds to new problems with the support of a professional staff of specialists.

\section{The true specialist}

Here we come again to the distinction between the specialist and the consultant. The true consultant is not a specialist; he is a generalist in specialist clothing, 
which he can change to suit the climate. The true specialist is the man who does a single job supremely well-the Smith, whose family motto "one thing well done" exemplifies his ambition.

There is much merit in the British Royal Commission Report's recommendation that three years after graduation should be spent in general professional training, which can be arranged on a modular basis to prepare people for different specialist careers, including general practice. This at least enables a man to fit himself for a job and take his place in the community; some will remain thus, and take pride in being true specialists; others will seek further experience, in depth and in breadth, to become consultants. This is not altogether a matter of superior and inferior beings; it is a matter of different kinds of people with different personalities and a different sense of proportion between doing, thinking, and organizing the work of others.

As specialist training becomes longer and more detailed the general aspects of education become compressed more and more into the early years. We are in danger, and we know it, of producing people who are good specialists but who know little else about how to enjoy life and appreciate what the world has to offer. It would be good if we could somehow reverse the natural order of things; one thinks of the quip about Hollywood people who live happily and get married ever after. If only we could get people established in their careers and then resume their general education! This is no easy problem to solve; the idea of professional education followed by consultant education is a step in the right direction as long as consultant means what it should, and not just a more specialized specialist.

\section{Our needs and world needs}

In residency training we deal not only with our own graduates but with many doctors who seek training from overseas. One finds wide differences in viewpoint among these foreign graduates. Their motives are various: some wish to train and settle in Canada; some seek training in order to return home; some do not declare their intentions, and some have not made up their minds. All this makes a difference, and this is not to say that the quality of training should be based on the principle of one law for the rich and one for the poor; it is an extension of the concept of residency training as one phase of an extensive process. How can we expect that a residency appropriate for specialist practice in Canada will fit the needs of India or Korea? As an affluent society we have something to offer in the way of graduate training and we must therefore accept an obligation to countries where the same training facilities may not be available. If we do accept this obligation it begs the question to complain about the quality of the undergraduate training of some of the people who come. A developing country may not have a very good medical school, but it may need doctors very badly and it may be producing the best doctors it can in the present circumstances. It may also be in desperate need of specialists, and its own admitted shortcomings make it all the more important for these potential specialists to come to us. There would be much to be said for establishing graduate training centres for overseas doctors whose declared intention is to return to their own countries. This is the best way we can hope to meet their special needs. 
Foreign graduates who intend or hope to remain in Canada should be accepted on their merits and be of the same standard as Canadian graduates. The first year of residency will often be a probationary period of assessment and this should be made clear. To make up the numbers in a residency programme by accepting foreign graduates more or less indiscriminately after places have been found for domestic applicants takes us back to the conflict of quality and quantity; it is evading the issue which it has been the purpose of this paper to discuss. 